\title{
Student Attendance Management System using Principal Component Analysis Method
}

\author{
M. Srilatha, R. Hemalatha
}

\begin{abstract}
Attendance taking and maintaining is a tedious job in the academic institutions where the time of class is restricted. The manual attendance i.e., roll call or paper-based signature systems usually consumes more time and error prone and also possibility of recording proxy attendance is more. Attendance is one of the criteria in considering students' eligibility for attending the external examinations and also for the promotion to the next semester / year, where these kinds of problems may cause severe effect on the academic institutions. As the strength of students in a class is increasing day by day; monitoring, awarding and maintenance of attendance has becoming a challenge for the academic institutions.

As a solution, attendance can be recorded using anyone of the existing biometric techniques like fingerprinting, iris recognition, signature, face recognition etc. Face identification is the best method among all the earlier mentioned methods for implementing in the academic institutions as it does not require human intervention and it is a cost-effective technique. A novel student attendance recording and management system using a MATLAB application, LabVIEW, Camera interface and GSM is proposed in this paper. Students' faces will be captured with the help of a camera connected to a computer and Eigen values of the captured images will be detected with the help of MATLAB executed by LabVIEW Mathscript node.

LabVIEW, a graphical programming environment is adopted for acquiring face, processing and authenticating the student once the match is found. Authenticated student attendance will be updated, and a message will be sent with the help of GSM module interface to myRIO. Proposed system replaces the manual attendance system which improves the performance of existing system.
\end{abstract}

Keywords: Image Processing, Face Recognition, myRIO, LabVIEW, MATLAB.

\section{INTRODUCTION}

$\mathrm{C}$ onventional way of recording is tedious and may not be completely accurate particularly in the case of larger size class. This method requires student's attention during the lecture and also consumes the valuable time of the faculty in roll call, maintenance of manual record of attendance, keep tracking the students. It is also difficult for faculty to maintain and monitor the attendance of students on regular basis. Automatic attendance systems are being used by most of the organizations for the purpose of recording attendance of the

Revised Manuscript Received on March 18, 2020.

* Correspondence Author

M. Srilatha*, Department of Electronics and Communication Engineering, Vardhaman College of Engineering, Hyderabad, India. Email: ma_srilatha2@yahoo.co.in

R. Hemalatha, Department of Electronics and Communication Engineering, University College of Engineering, Osmania University, Hyderabad, Telanagan, India. staff / students. Some of the existing systems are based on IRIS [1], FINGERPRINT [2], [3], RFID [4], [5], IBeacon [6], Bluetooth [7] etc. The majority of these systems demands more time in marking the attendance, resulting in even maintaining queue sometimes and these systems are intrusive in nature. Though quick marking of attendance can be achieved up to some extent, RFID card damage may result in inappropriate attendance. Biometric [8] attendance system based on fingerprint may not effectively work during winter and also few persons fingerprints may not be captured by the receiver properly; on the other hand, IRIS based biometric attendance systems [1] need more amount of time to read. Apart from all the above limitations the major drawback of the existing systems is it is not cost effective to implement on large scale for organizations like educational institution where thousands of members need to mark their attendance within limited time. Maintaining the Integrity of the Specifications.

The rapid growth in the proposed field progressed in introduction of pattern recognition, facial recognition [9], [10], [11], [12] and signature recognition with increasing efficiency in recognition [13]. The proposed system automates attendance system using image / video stream [14] in recording the attendance in a class while keeping the database of the attendance. Once the data base is created, the proposed system needs almost zero efforts from the user end. It is based on facial recognition which is primary identification of any person which is a cost effective, requires very less amount of time compared to the other existing systems, don't demand much human intervention and does not requires the cooperation of the test subject. The rapid changes in the society in adopting advanced technology in day to day life demands the security measures and proper identification. The proposed technology uses the most common yet unique identification method of using face recognition in many applications and has a great scope of research.

The proposed method basically depends on face detection at the initial phase. There are a numerous existing algorithm with difference in response time, accuracy and efficiency. Each algorithm uses a particular method, Principal Component Analysis (PCA) [15], [16] algorithm uses eigen faces created by the convolution and major features of the faces of the database. PCA allots a specific weight to each face to compare with the Euclidean distance. The Discrete Cosine Transform (DCT) [17] algorithm expresses various data points in terms of a sum of cosine functions at different frequencies; Fisher face method makes the use of Linear Discriminant Analysis (LDA) [18], 
[19] is another variation to eigenfaces which has an advantage of recognizing the face at deferent variations in illumination [20]. All of the face recognition algorithms/techniques are based on the calculation of a set of geometrical features from the picture of a face.

The proposed system uses MATLAB and LabVIEW together [21], [22] to build a software program for recognizing human faces. It combines the advantages of LabVIEW and MATLAB. Math Script node is used in LabVIEW [23], [24], [25] to compile and execute MATLAB code. It can detect and recognize the face in real time and has the advantages of simple structure, user friendly interface, easy to use.

\section{A. Outline}

The paper is organized as follows: Section 2 discusses about detailed methodology design of the proposed system. Section 3 explains the flowchart of the system. Section 4 shows the simulation results. The last section is about conclusion of the work and future scope.

\section{METHODOLOGY'}

Block diagram of the proposed system is depicted in Fig 1. The proposed system functioning depends on the face recognition using LabVIEW which marks the students' attendance automatically. Each student attendance will be updated as present once his/her face pattern is analysed with the help of input images from the camera. Each student image is initially stored in train data base which is further used to identify the student.

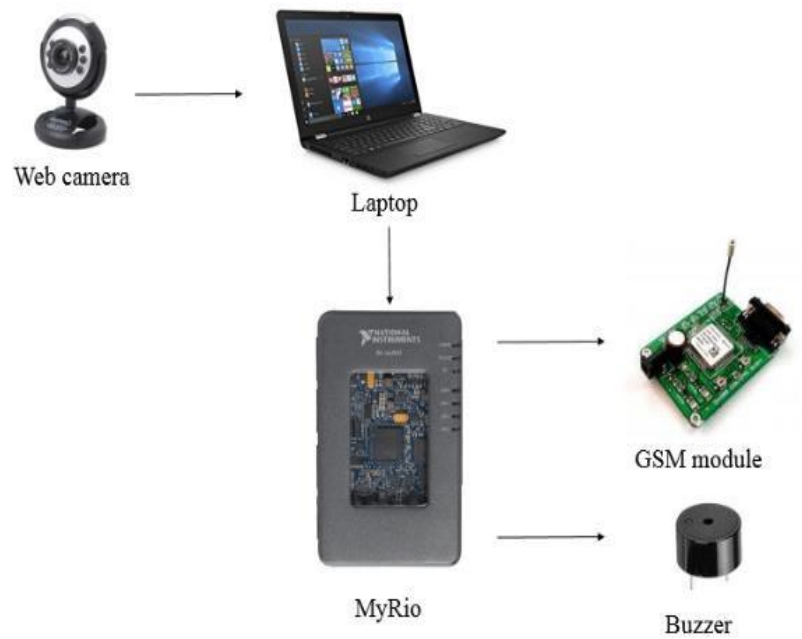

Fig 1: Block Diagram of proposed system

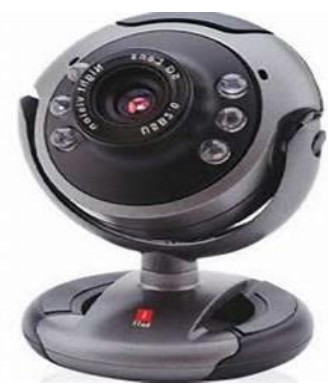

Fig 2: Web Camera

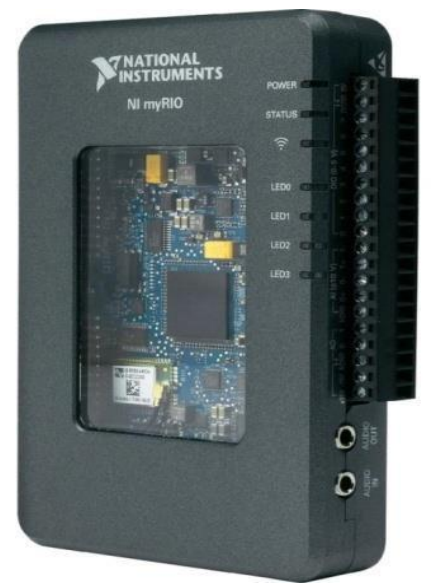

Fig 3: NI myRIO

NI myRIO process the input image from the camera by comparing the test database and verifies the train database for matching. Once the face pattern matches, the buzzer will buzz to indicate student's attendance marking as present and further a message will be broadcasted to the parent informing the presence of the student. The proposed system uses NI myRIO, Webcam, GSM module and a Buzzer whose description is mentioned below

\section{A. Webcam}

Webcam used in the proposed system as shown in Fig 2 is a video camera use to feed and streams its output in the form of images in real time using a laptop. Unlike IP camera, a webcam can be easily connected to a laptop or a computer with USB cable which is available for very less price in the market.

\section{B. NI myRIO}

Fig 3 shows NI myRIO, a product of National Instruments, is a real time embedded evaluation board. The onboard FPGA and microprocessor of myRIO are used to develop applications with the help of LabVIEW software to control myRIO. It has $667 \mathrm{MHz}$ dual-core ARM Cortex-A9 programmable processor and a customizable Xilinx Field Programmable Gate Array (FPGA). myRIO has both analog and digital input and outputs along with audio and power output which can be connected to a host computer using USB cable or WiFi.

\section{GSM Module}

Global System for Mobile communication (GSM) module as shown if Fig 4 is a modem to broadcast messages in the proposed system. Time Division Multiple Access (TDMA) technique was used in the GSM module to transmit mobile voice and data operating at frequencies $850 \mathrm{MHz}, 900 \mathrm{MHz}$, $1800 \mathrm{MHz}$ and $1900 \mathrm{MHz}$ and the module has the ability to carry data at $65 \mathrm{Kbps}$ to $120 \mathrm{Mbps}$ speed. The TDMA technique digitizes and reduces the data and then transmits through a channel with two different streams of client data, each in its own particular time slot. 


\section{Buzzer}

Buzzer shown in below Fig 5 is an alerting component mostly used in the domestic devices like washing machines, microwave ovens, refrigerators etc. It is connected to a control unit which passes signal to the buzzer as and when the student's attendance is marked or failed to recognize. Up on receiving the signal the buzzer will make the intermittent or continues beeping or buzzing sound to alert the student.

In the proposed system an external webcam is connected to the laptop for high resolution image acquisition using vision toolkit. Also, NI myRIO is connected to the laptop using USB cable to use myRIO as control unit. The connection diagram of the proposed system is shown in the below Fig 6 .

\section{FLOWCHART}

The Flow chart of the proposed system is depicted in the Fig 7. It is initialized by setting all controls and indicators on the front panel to default values. A student's face will be acquired from the webcam for either recognizing or storing into the database. The image is processed with the help of VI's which are predefined or defined by the user. If the student's face is captured for acquisition, the image will be stored in the desired location else, if the image is captured for face recognition, it will be stored in a temporary location called as Test Database.

The Test Database provides the input (stored images) during the recognition process. During the recognition process, the Eigen values of the captured image will be generated along with the Eigen values of each image in the Train Database. After generation of Eigen values, the captured image values are compared with the Eigen values of each and every image of the Train Database. Using Vectorization process Eigen vectors of all the images in Train Database are converted from $2 \mathrm{D}$ matrix of images to $1 \mathrm{D}$ matrix.

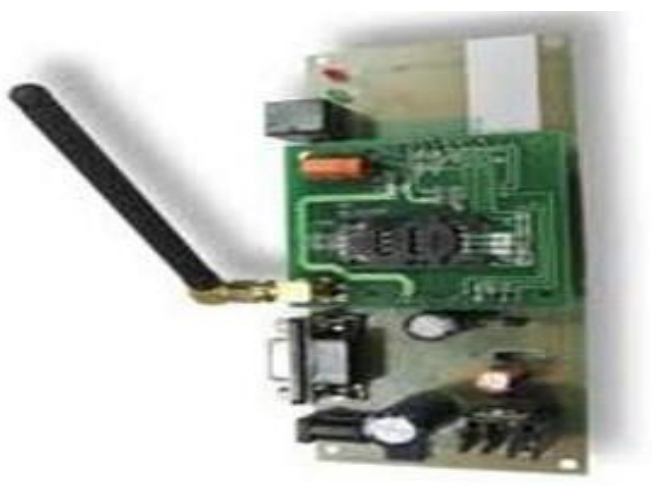

Fig 4: GSM Module

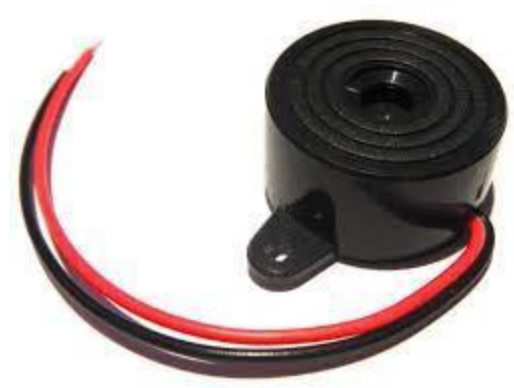

Fig 5: Buzzer

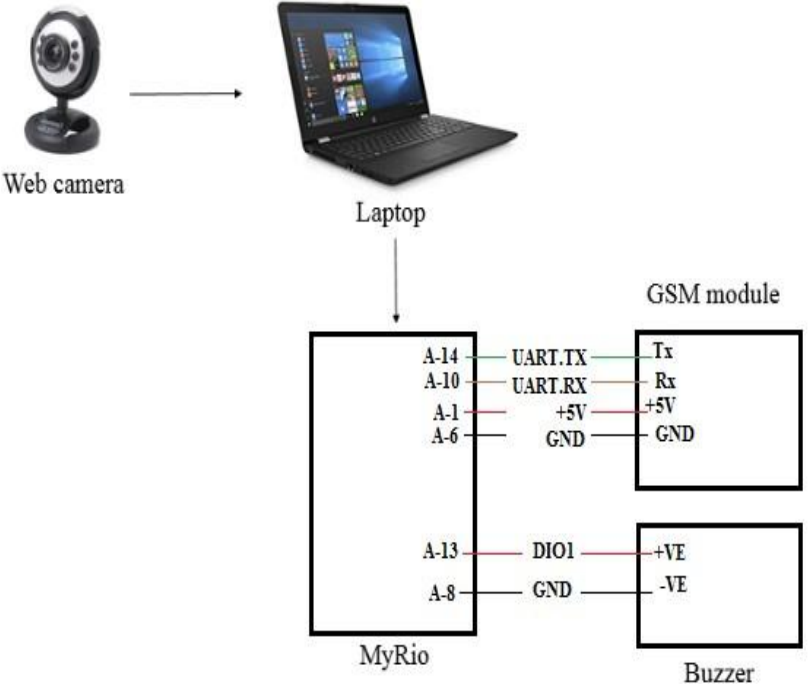

Fig 6: Connection diagram of proposed system

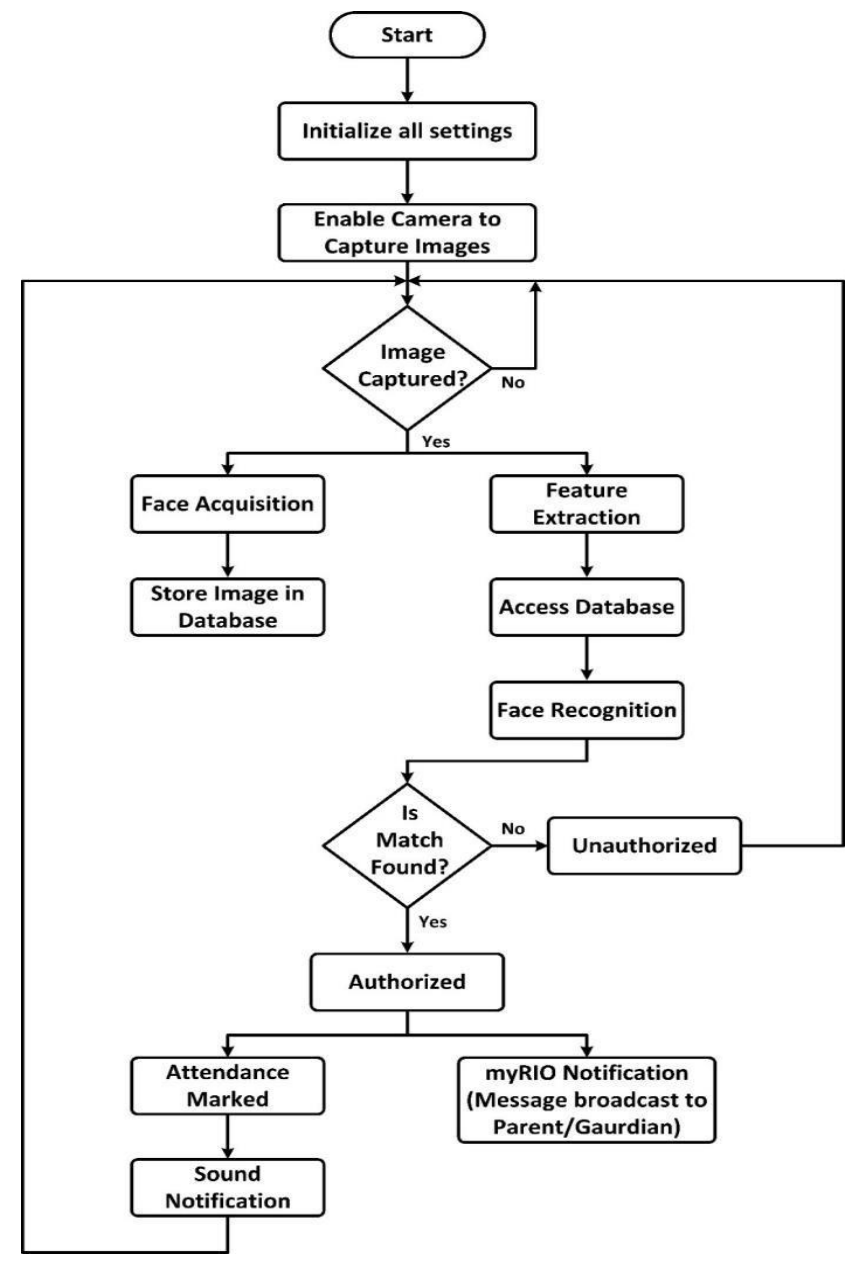

Fig 7: Flowchart of proposed system

MATLAB is used for Vectorization and face recognition, in the form of Math Script Node in LabVIEW. Once the Eigen values of captured image and Eigen values of any one of the train database images matches within a threshold limit, the contact details like mobile number or an Email id linked to the matched image are extracted and a message is broadcasted confirming the attendance of the student to the parent. 
Once the attendance of a student is marked, the buzzer will produce beep sound to notify the same to the student. Further, the system will capture the next student's face and repeat the same process.

The best part of the proposed system is the memory management. If the flow of the images is too high, all these temporary images can be stored in the test database and once the flow settles, all these temporary images will undergo process of recognition one by one.

\section{RESULTS \& DISCUSSION}

Fig 8 depict the front panel of the proposed system which is simulated using LabVIEW software. Inputs and Outputs are displayed as icons.

The complete process of Proposed system is divided into five states:
A. Waiting State.
B. Acquisition State for Train Database.
C. Acquisition for Test Database.
D. Image Recognition State.
E. Message State.

\section{A. Waiting State}

The initial state of the proposed system is waiting state where the system waits for student image as shown in Fig 9. Once the image is captured by the webcam then the system will move from waiting state to next state.

\section{B. Acquisition State for Train Database}

The captured image will be stored in the train database, where two images of each student are stored which are used to identify the person whose attendance has to be registered as shown in Fig 10. Once the student roll number is entered and acquisition save button clicked, the student photo will be saved with the roll number in the train database. The proposed system has an advantage of capability to store more than two images of a particular student in the train database, which is helpful in providing more précised output. Once the images is stored, the system will switch to either waiting state or any of the other state using enum local variable as shown in Fig 11.

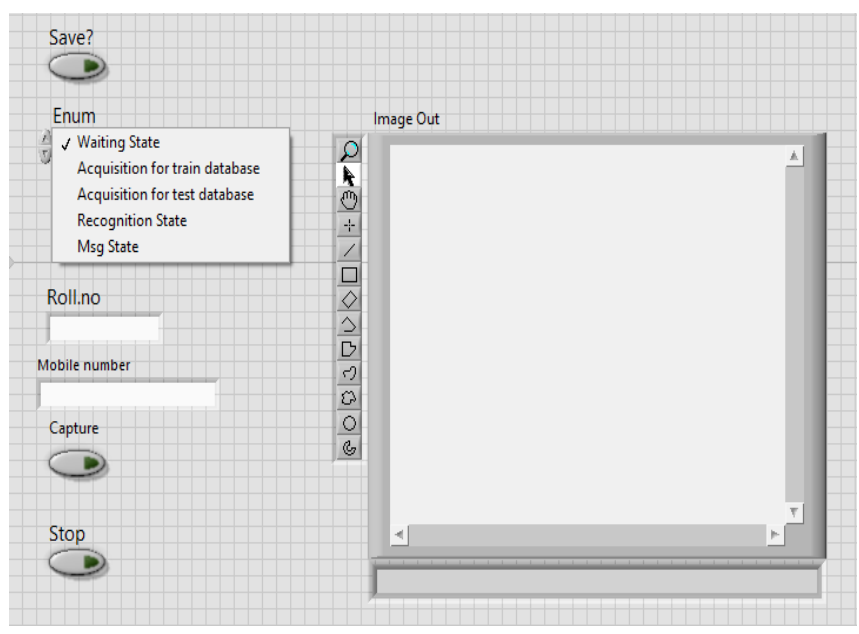

Fig 8: Front panel of the proposed system

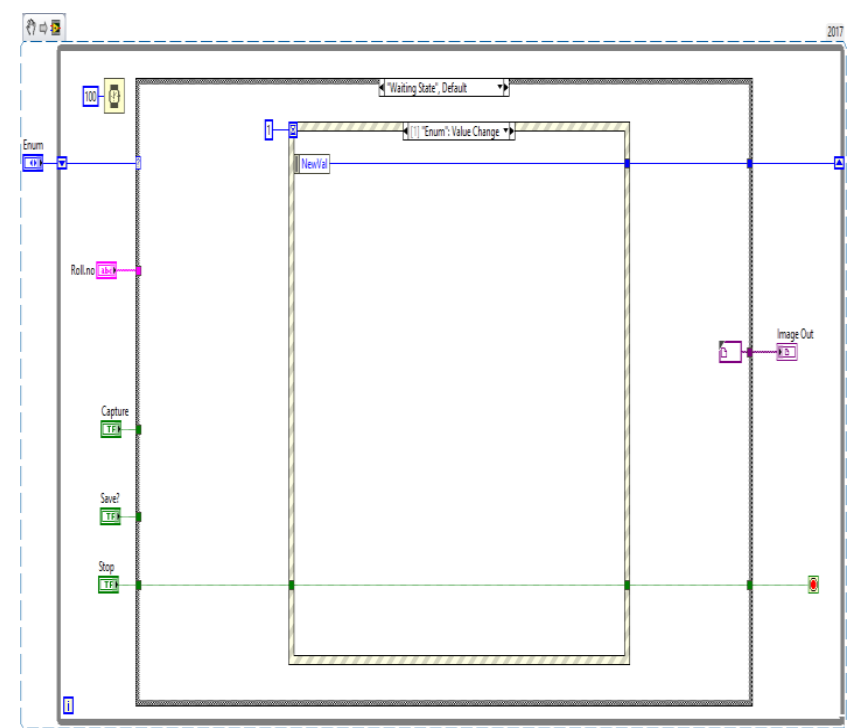

Fig 9: Block Diagram of Waiting State

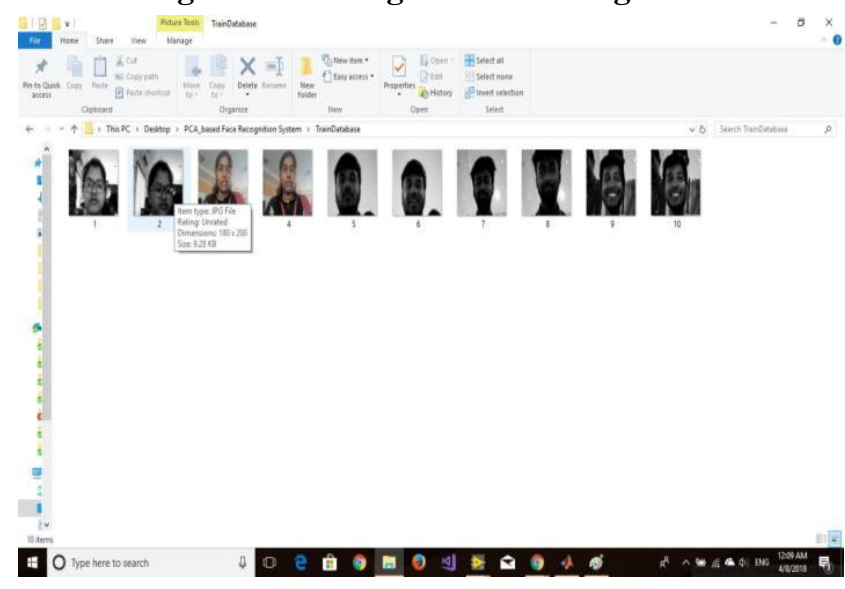

Fig 10: Images in Train Database

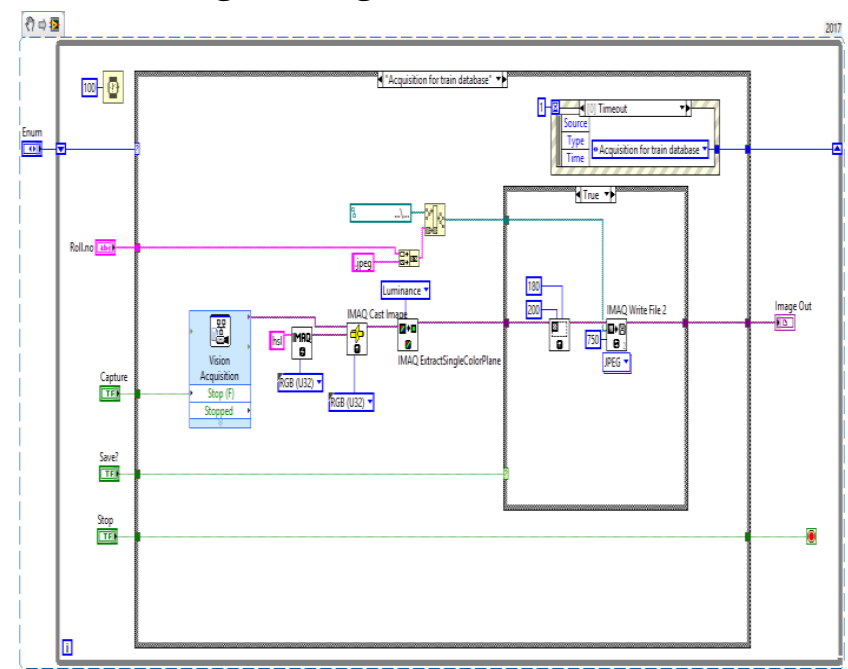

Fig 11: Block Diagram of Acquisition State for Train Database

The image can be directly stored into Train database. However, to get more accurate results, Vision acquisition is used to acquire images and then converts into black and white image. 


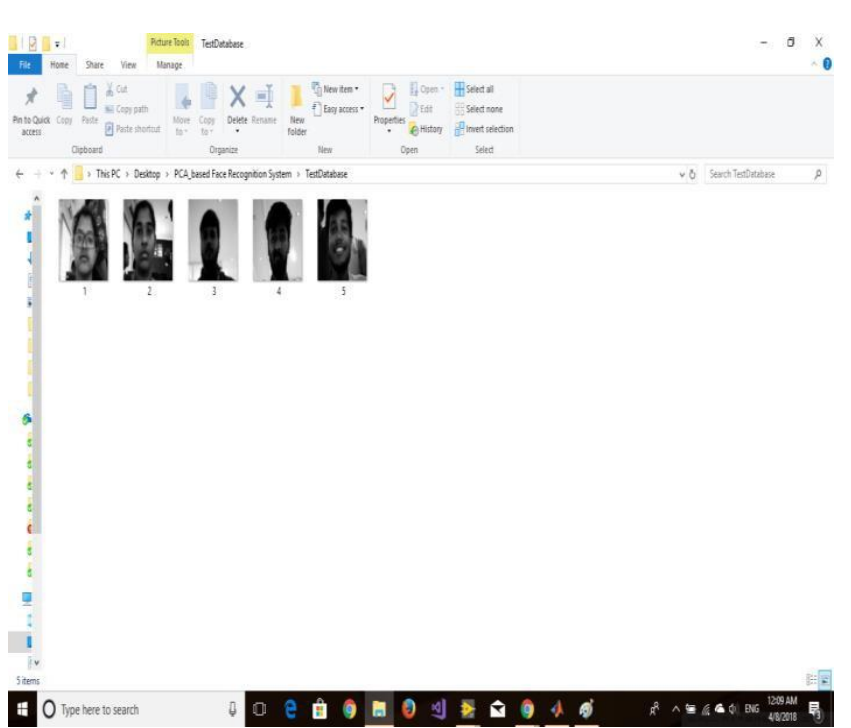

Fig 12: Images in Test Database

\section{Acquisition State for Test Database}

In this state, image is acquired as same as in capturing for train database by using vision acquisition to acquire images. But the difference is that only one image is saved into test database from a steam of acquired images using recognition save button. Test database is the database which contains images that has to be compared with train database for recognition as shown in Fig 12.

The advantage of the test database is, if the recognition is failed due to any error in the recognition process, the image stored in the test database can be used without causing any problem as shown in Fig 13.

\section{Image Recognition State}

In image recognition state as shown in Fig 14, a popup window will be displayed to choose the image stored from the test database that has to recognize and the recognition part is implemented using MATLAB. Once the image name is entered, it scans through the train database that matches the image. If the matching image is recognized, then test image and matched image pops up and attendance of that particular student will be marked as present and a buzzer gives small sound as signal indicating recognition is done. Once recognition is done, it moves onto the message state.

\section{E. Message State}

Once the face recognition is done, a message will be broadcasted to the concerned parent / guardian / student whose mobile number is stored through the front panel mobile number control. A GSM module is used to send the message as shown in Fig 15. Once the message is broadcasted, the system will go to the waiting state to wait for the new user to come. Block diagram of message state is shown in Fig 16. Prototype of the proposed system is shown in Fig 17.

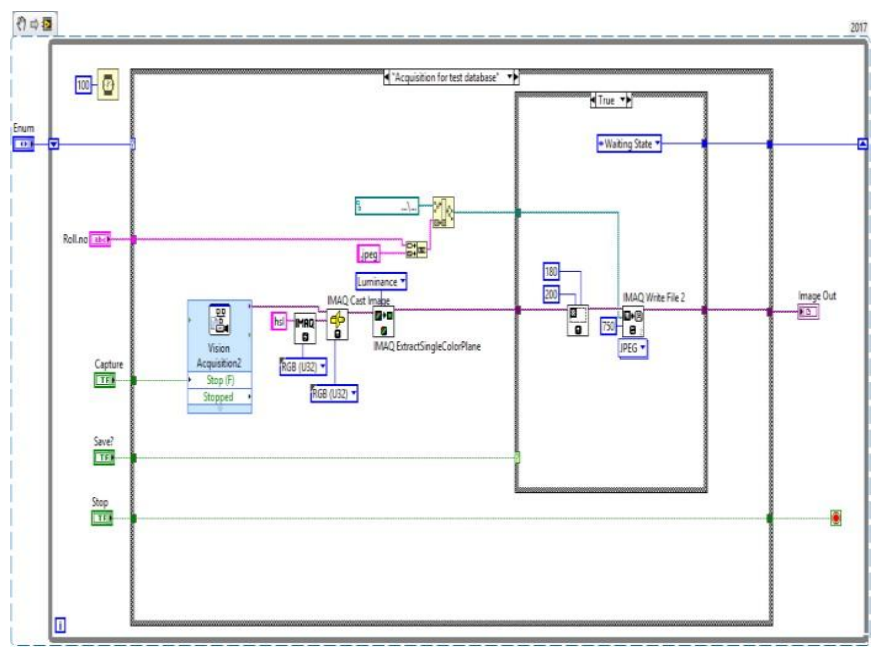

Fig 13: Block Diagram of Acquisition State for Test Database

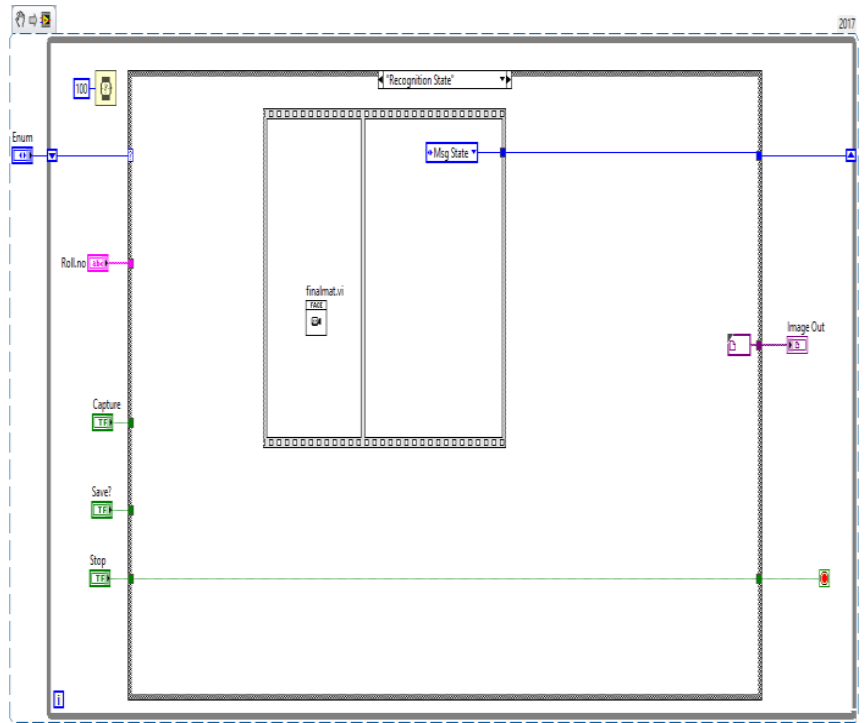

Fig 14: Block diagram of Image Recognition State

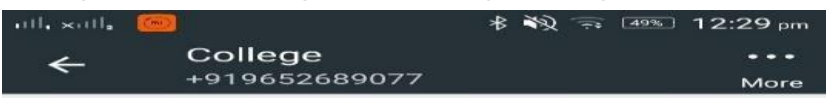

Your ward has attended the college

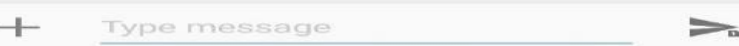

Fig 15: Message send to the desired person 


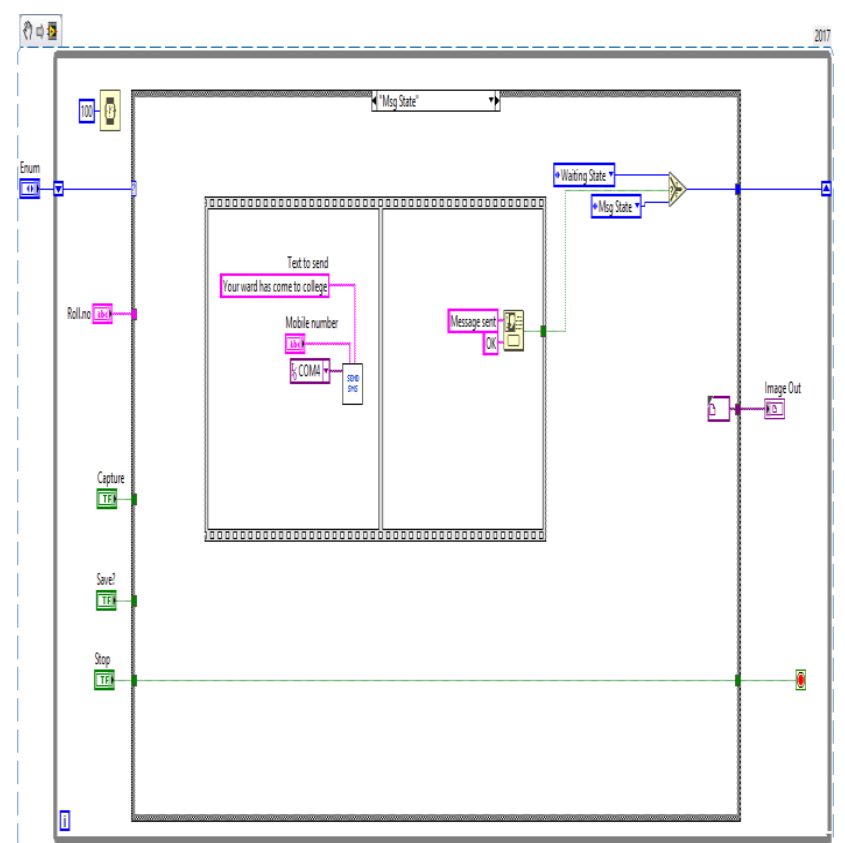

Fig 16: Message State

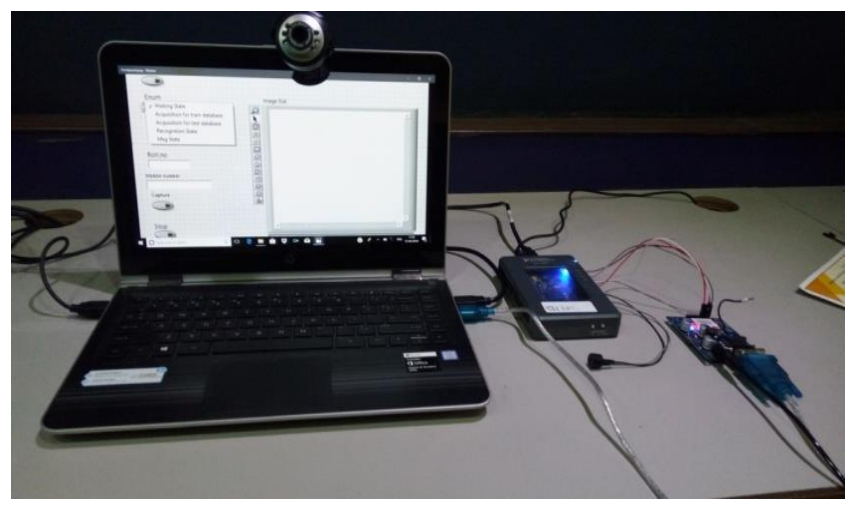

Fig 17: Prototype of proposed system

\section{CONCLUSION \& FUTURE SCOPE}

To overcome the problems in conventional attendance recording by means of roll call during the lecture, an automatic attendance management system based on face detection and face recognition technique using NI LabVIEW with vision toolkit and MATLAB software is proposed. The Proposed system increases the performance of existing system in automatic tracking and recording of a student attendance, reduce the time required for recording the attendance, accuracy in marking the attendance of a student, broadcasting the message to a parent / guardian / student about the student presence, increase the overall efficiency of the system and improve the security. The system can also be optimised in future as an unobtrusive audio and video-based student identification system which can achieved high recognition rates without requiring the user to be in highly controlled environment.

\section{REFERENCES}

1. Rupali L. Telgad and Alams Siddiqui (2017), "Development of an efficient and secure biometric system by using Iris recognition", International Conference on Intelligent systems and Information management (ICISIM), Vol. 6, pp. 222-270.
2. Le Hoang Thai ang Ha Nhat Tan (2010), "Fingerprint Recognition using standardized finger print model", International Journal of Computer Science issues, Vol. 7, Issue. 3, pp. 7.

3. Y. Mittal, A. Varshney, P. Aggarwal, K. Matani, and V. K. Mittal "Fingerprint biometric based access control and classroom attendance management system," in 2015 Annual IEEE India Conference (INDICON), pp. 1-6, New Delhi, India, December 2016.

4. S. Pss and M. Bhaskar, "RFID and pose invariant face verification based automated classroom attendance system," in 2016 International Conference on Microelectronics, Computing and Communications (MicroCom), pp. 1-6, Durgapur, India, January 2016.

5. T. Lim, S. Sim, and M. Mansor, "RFID based attendance system", in Industrial Electronics \& Applications, 2009. ISIEA 2009. IEEE Symposium on, vol. 2. IEEE, 2009, pp. 778-782

6. Muhammad Ayat Hidayat, Holong Marisi Simalango, "Students Attendance System and Notification of College Subject Schedule Based on Classroom using IBeacon", 2018 3rd International Conference on Information Technology, Information System and Electrical Engineering, 13-14 Nov.2018.

7. Rishi Raj, Abhinav Das, Subhash Chand Gupta, "Proposal of an Efficient Approach to Attendance Monitoring System using Bluetooth”, 2019 9th International Conference on Cloud Computing, Data Science \& Engineering, 10-11 Jan 2019.

8. H. Adal, N. Promy, S. Srabanti, and M. Rahman, "Android based advanced attendance vigilance system using wireless network with fusion of bio-metric fingerprint authentication," in 2018 20th International Conference on Advanced Communication Technology (ICACT), pp. 217-222, Chuncheon-si Gangwon-do, Korea (South), Febuary 2018.

9. Kennedy Okokpujie, EtinosaNoma-Osaghae, Samuel John and KaluAnyah Grace (2017), “A Face Recognition Attendance System with GSM notification”, pp. 239-244.

10. C S Patil, R RKarhe and M D Jain (2014), "Student Attendance System and Authentication using Face Recognition", International Journal of Engineering Research \& Technology, Vol. 3, Issue. 7, pp. 373-375.

11. NazareKanchan Jayant, SurekhaBorra(2016), "Attendance Management System using Hybrid Face Recognition Techniques", Cinference on Advances in Signal Processing (CASP), pp. 412-417.

12. Shubrobrata Bhattacharya, Gowtham Sandeep Nainala, Prosenjit Das, Aurobinda Routray, "Smart Attendance Monitoring System (SAMS): A Face Recognition Based Attendance System for Classroom Environment", 2018 IEEE 18th International Conference on Advanced Learning Technologies, 9-13 July 2018.

13. Sonam Shukla, Pradeep Mishra (2012)," Increasing the accuracy of an existing recognition system using adaptive technique", International Journal of Advanced Research in Computer Science and Engineering, Vol. 2, Issue. 6, pp: 52-57.

14. S. Matilda, K. Shahin, "Student Attendance Monitoring System using Image Processing”, 2019 IEEE International Conference on System, Computation and Networking, October 2019.

15. Aalam Gumber, Navneet Kaur, "Face Recognition Based Automated Attendance Management System using Principal Component Analysis", International Journal of Science and Research, Vol. 4, Issue. 6, pp. 971- 975, 2015.

16. Akinrotimi, Akinyemi Omololu, Dayo Reuben, "Facial Emotion Recognition using Principal Component Analysis and Support Vector Machine", Journal of Computing, Information Systems, Development Informatics \& Allied Research, Vol. 9, Issue. 2, pp. 12-20, June 2018.

17. Samuel Lukas, Aditya Rama Mitra, Ririn Ikana Desanti, Dion Krisnadi, "Student Attendance System in Classroom using Face Recognition Technique", International Conference on Information and Communication Technology Convergence, 2016.

18. Kritika Shrivastava, "Conceptual Model for Proficient Automated Attendance System based on Face Recognition and Gender Classification using Haar-Cascade, LBPH Algorithm along with LDA Model", International Journal of Applied Engineering Research, Vol. 13, Issue. 10, pp.8074-8080, 2018.

19. V. Kurniawan, A. Wicaksana, and M. I. Prasetiyowati, "The implementation of eigenface algorithm for face recognition in attendance system," in 2017.

20. S. Yang, Y. Song, H. Ren, and X. Huang, "An automated student attendance tracking system based on voiceprint and location," in 2016 11th International Conference on Computer Science \& Education (ICCSE), pp. 214-219, Nagoya, Japan, August 2016. 
21. Wunixu, LanxiangZhong, Dingyuan Wang, "Image Processing Based on Seamless Integration Technology Between Lab VIEW and MATLAB", IEEE International Conference on Information,Networking and Automation (ICINA), pp. 219-223, 2010.

22. M. Srilatha, R. Hemalatha, "A Novel Robotic Arm Design for Small Scale Industries using myRIO", International Journal of Engineering \& Technology, Vol. 7, No 4.6, 2018.

23. Amit Kumar, P K Verma, Srinivas Perala and P R Chandha (2016), "Automatic Attendance System by Visual Programming Language LabVIEW", IEEE conference on Power Electronics, Intelligent Control and Energy Systems.

24. Sudha Rani K, T C Sarma and K Satya Prasad (2013), "Face Recognition Office Security System using LabVIEW 8.6", International Journal of Electronics Communication \& Instrumentation Engineering Research and Development, Vol. 3, Issue. 2 pp. 195-200.

25. Jerome, Jovitha, "Virtual Instrumentation using LabVIEW" 1stEdition, PHI, 2010.

\section{AUTHORS PROFILE}

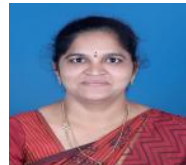

M. Srilatha received her B.Tech in Electronics and Communication Engineering from Vijay Rural Engineering College, Nizamabad, Telangana in 2004 and her M.E degree in Digital Systems Engineering from Osmania University, Hyderabad, Telangana in 2010. She is currently pursuing $\mathrm{PhD}$ in Wireless Communications from Osmania University. Her research interests include low latency high throughput spectrum sensing, Cognitive Radio, Robotics. Till now she published more than 10 papers including international journals and conferences. She is having memberships in IEEE, IAENG, SAISE, UACEE and IET.

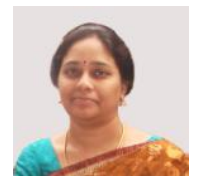

Dr. R. Hemalartha completed her PhD from JNTUH University in the area Wireless Communication in 2012. She is currently working as Associate Professor in Department of Electronics and Communication Engineering, University College of Engineering, Osmania University. She has a number of publications and also successfully completed the project sponsored/funded by AICTE. 